\title{
OSTEOMYELITIS OF THE MAXILLA IN INFANCY
}

BY

\author{
J. C. HAWORTH
}

(From the Children's Hospital, and the Department of Child Health, Birmingham)

Acute suppurative infection in infancy is of peculiar interest in the special clinical features according to (a) the bacteriology, and (b) the localization. Osteomyelitis is by no means a rarity in the early months of life. The staphylococcus is a prevalent micro-organism, and its mode of entry into the tissue is often difficult to trace; but fortunately a prompt cellular defence is rapidly mobilized, and, with the advent of chemotherapy and antibiotic remedies, recovery from such infection is now very much more hopeful. Acute osteomyelitis of the maxilla is an example; it may occur in the early weeks of infancy. During the past fifteen months five cases of this condition have been admitted to the Children's Hospital, Birmingham, and this short paper is a record of their progress.

The local anatomy is of some interest; the antrum is present at birth, and the superior maxilla is a highly vascular area of diploic bone, having as its upper boundary the lower border of the orbit, and extending down to the dental ridge containing the primordial follicles in which are embryonic teeth enclosed within a vascular bed. The infecting organism may gain entrance to the superior maxilla in a variety of ways: (1) from the adjacent maxillary antrum, which in infancy is always very prone to infection; (2) from the lachrymal duct and sac; (3) from a primary infection of the embryonic teeth follicles, which may occur from the blood stream or by trauma to the overlying gum occurring at, or soon after, birth. It is in the latter way that infection occurred in two of the following cases, as the infected dental radicle was removed surgically. This of course disturbs the primary dentition, and occasionally the permanent dentition may also be affected.

Jacoby and Sagorin have recently reviewed the subject and recorded the favourable progress in three cases. Their commentary on the treatment is important, especially in regard to the desirable minimal surgical attention, bearing in mind that the dental areas may suffer interference; also cosmetic effects must be'kept in mind. These authors have noted the remarkable and rapid effects of penicillin in this particular disease; healing was obtained in one or two weeks, as compared with an illness of months' duration and high mortality with former lines of treatment.
Case Reports

Case 1. A male baby of four weeks, breast-fed, was admitted to hospital because three days previously the mother had noticed a swelling on the left side of his face and over the eye. There appeared to be some discharge from the eye and the nose on the same side. No diarrhoea or vomiting was present.

EXAMINATION. The baby weighed $5 \mathrm{lb} .9 \mathrm{oz}$. There was proptosis of the left eye, chemosis, and some purulent discharge from the lachrymal duct. The left cheek was reddened and markedly swollen, and gentle pressure caused a purulent discharge to come from the left nostril. A small sinus was discharging in the left alveolus. Temperature was $101^{\circ} \mathrm{F}$., and the pulse rate 148 per minute.

Treatment. Penicillin, 1,500 units three-hourly, was given intramuscularly, and sulphadiazine, $0.4 \mathrm{~g}$., followed by $0.2 \mathrm{~g}$., six-hourly. The baby's condition improved, and forty-eight hours later a small incision was made over the left alveolus and a diseased tooth follicle was removed. A large amount of pus was evacuated, and the swelling appeared to diminish in size and become much less tense. Culture of the pus revealed a heavy growth of staphylococcus aureus. In a further forty-eight hours there was a vast improvement, and penicillin treatment ceased. In still another forty-eight hours the swelling of the face had disappeared; the baby could open and close the eye. Twenty-four hours later there was slight recurrence of swelling in the cheek below the left eye, and another twenty-four hours' course of penicillin was given, with the result that the signs of local infection completely subsided and the baby was discharged clinically well six weeks after admission. A total of 120,000 units of penicillin was given in the first course, and 156,000 units in the second course.

Case 2. A male baby, aged four weeks, had been ill for ten days before admission to hospital, the parents having noticed a swelling below the left eye for several days.

EXAMINATION. A small dehydrated infant, weighing $7 \mathrm{lb} .9 \mathrm{oz}$., had a firm smooth swelling in the left side of the face which seemed to involve the whole maxilla. This swelling extended into the mouth and the palate on the left side, where there was a small discharging sinus. There was marked conjunctivitis and chemosis on the same side. No diarrhoea or vomiting was present.

TreatMENT. On the date of admission free 
drainage was established by opening into the left side of the palate and alveolus. Thick pus was released. Culture showed haemolytic staphylococcus aureus, and a few pneumococci. Intramuscular penicillin, 1,500 units three-hourly, was given over a period of five days, and penicillin was applied locally for a few days. The inflammatory swelling quickly subsided; the incision healed, and rapid clinical progress was made. The baby was discharged from hospital after a stay of fifteen days. A total dose of 96,000 units of penicillin was given over eight days.

Case 3. A male baby, aged seven weeks, who had been artificially fed, had had a septic finger during the first week of life, and a swelling below the left eye had developed ten days before admission to the Children's Hospital. The child was referred from the Birmingham and Midland Eye Hospital with a history of a left-sided nasal discharge and swelling of the left cheek and left upper alveolus. Penicillin had been given, and an incision made into the lower lid.

EXAMINATION. The baby was well nourished, weighed $7 \mathrm{lb} .12 \mathrm{oz}$., and had a temperature of $100^{\circ} \mathrm{F}$., and a pulse rate of 130 . There was a swelling under the left lower lid, and pressure over this area caused a blood-stained serous discharge to appear from the nostril. There was no diarrhoea or vomiting.

TREATMENT. An incision was made over the left upper alveolar labial fold, and thick pus evacuated. Cultures gave a heavy growth of staphylococcus aureus. Several small sequestra were removed. From the lateral nasal wall and the region of the zygoma one infected dental radicle was enucleated. Intramuscular penicillin was continued, 1,500 units, three-hourly, for six days. The baby made favourable progress and the swelling rapidly subsided. After seventeen days' treatment in the Children's Hospital the baby was discharged home clinically recovered and gaining weight satisfactorily.

Case 4. A male baby, aged twelve weeks, had been breast-fed for one month, and weighed $11 \mathrm{lb} .7 \mathrm{oz}$. Twenty-four hours before admission he became extremely frefful, and a few hours later it was noticed that the left eye was swollen, and this gradually became worse during the day. There was no diarrhoea or vomiting.

EXAMINATION. The baby was pale but well nourished, with marked oedema and swelling about the left cheek and eyelid; the left eye appeared partly proptosed and the conjunctival sac purulent. There was a sinus discharging thick pus in the upper left alveolar region, and also a purulent discharge from the left nostril.

Treatment. Penicillin, 20,000 units, was given intramuscularly three-hourly; this was later reduced to 8,000 units. Sulphadiazine was also given. On the same day the alveolus was opened up a little and pus allowed to escape, and the diseased tooth radicle was removed. Culture of the pus grew haemolytic staphylococcus aureus. In forty-eight hours the swelling diminished; the eyelids were still very oedematous. Temperature became normal, and forty-eight hours later the swelling practically disappeared though it recurred slightly round the eye two days later, and a course of 20,000 units of penicillin was again given for forty-eight hours. The baby was discharged from hospital seventeen days from the date of admission; he was clinically well. (See Plate III.)

Case 5. A female baby, aged fifteen weeks, had previously been well and, fed on a milk and water mixture, had reached the weight of $14 \mathrm{lb}$. $5 \mathrm{oz}$. A few days after the baby had had a 'cold' some swelling of the left face and eye was noticed.

EXAMINATION. A well-nourished infant, with obvious proptosis of the left eye and gross oedema spreading down to the lower part of the face, had signs of bronchitis, a temperature of $103^{\circ} \mathrm{F}$., a pulse rate of 160 , and respiration rate 60 . Osteomyelitis of the left maxilla was diagnosed.

Treatment. Penicillin, 10,000 units three-hourly intramuscularly, and sulphadiazine $0.5 \mathrm{~g}$. six-hourly orally, were given on admission, and the general condition began to improve. After three days there was less oedema of the face, but a small incision was made in the nasal side of the maxilla and purulent fluid was evacuated. Radiographs showed that the ethmoids were dim. After eight days- of treatment the temperature subsided and the swelling was much less. The baby's condition was poor, and there were frequent watery stools. Three days later vomiting occurred and became severe, and deterioration set in with rapid loss of weight. Signs of nasopharyngitis and otitis media appeared. Bilateral myringotomy was performed but no pus was obtained. Intravenous fluid supplements were given, and $50 \mathrm{c.cm}$. of fresh blood transfused. Unfortunately the 'gastro-enteritis' persisted. A few days later right mastoidectomy was performed under local anaesthesia. The general state deteriorated further and death occurred thirty days from the beginning of the illness. Post-mortem examination showed left mastoiditis and haemorrhage, and toxic fatty changes in the liver and myocardium.

\section{Discussion}

The bacteriology in these cases was essentially that of a haemolytic staphylococcus aureus. An infected tooth radicle was removed in two cases, and it was in this infected radicle that the infection presumably started, having been carried there probably by the blood stream, as suggested by Jacoby and Sagorin (1945).

With the exception of one case (which was complicated by mastoid infection and a generalized toxaemia giving rise to enteritis and fatty changes in the liver and heart, and which eventually proved fatal) the infection was essentially a local one and was effectively cured by chemotherapy and a minimum of surgery to create free drainage. 
In view of the known susceptibility of infants of this age to develop gastro-enteritis in conjunction with parenteral infections, it is interesting that in all the cases excepting the fifth there was no diarrhoea or vomiting. This bears out the impression that the most obvious and frankly purulent infections in infancy, such as osteomyelitis, empyema, etc., do not tend to give rise to such severe alimentary disturbances as the more concealed neonatal infections of the middle ears, sinuses, mastoid antra, and umbilicus.
I am indebted to the Physicians and Surgeons of the Children's Hospital, Birmingham, for permission to publish these cases, and to Dr. A. V. Neale and Mr. Stirk Adams who have kindly reviewed this paper.

\section{REFERENCE}

Jacoby, N. M., and Sagorin, L. (1945). Arch. Dis. Childh., 20, 166. 


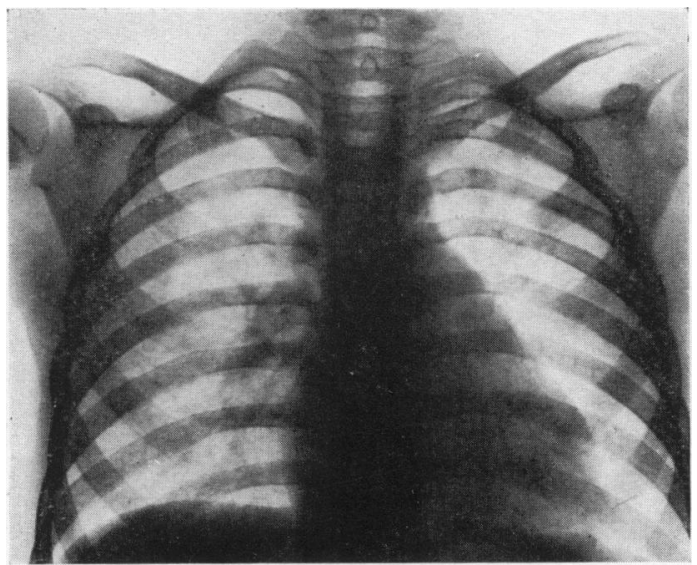

(j).--Antero-posterior view showing obstructive emphysema of the left lower lobe, with return of mediastinium to the normal position (case 6).

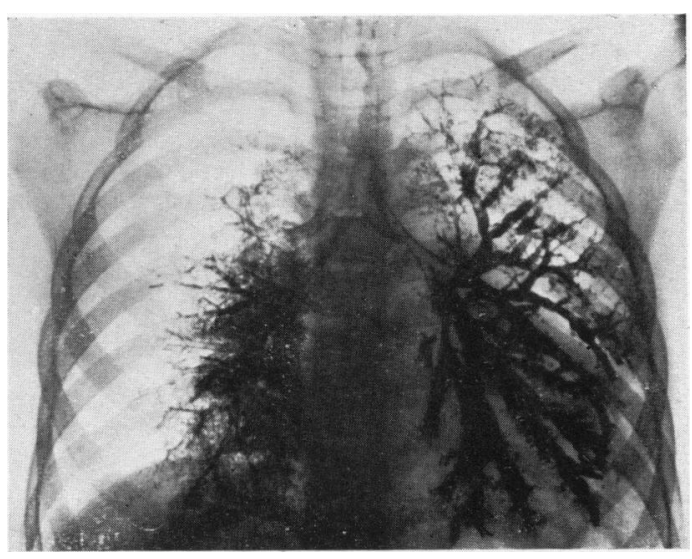

(k).- Showing cylindrical bronchiectasis of the left lower lobe, with narrowing of the left main bronchus (case 6).

\section{OSTEOMYELITIS OF THE MAXILLA IN INFANCY BY J. C. HAWORTH}

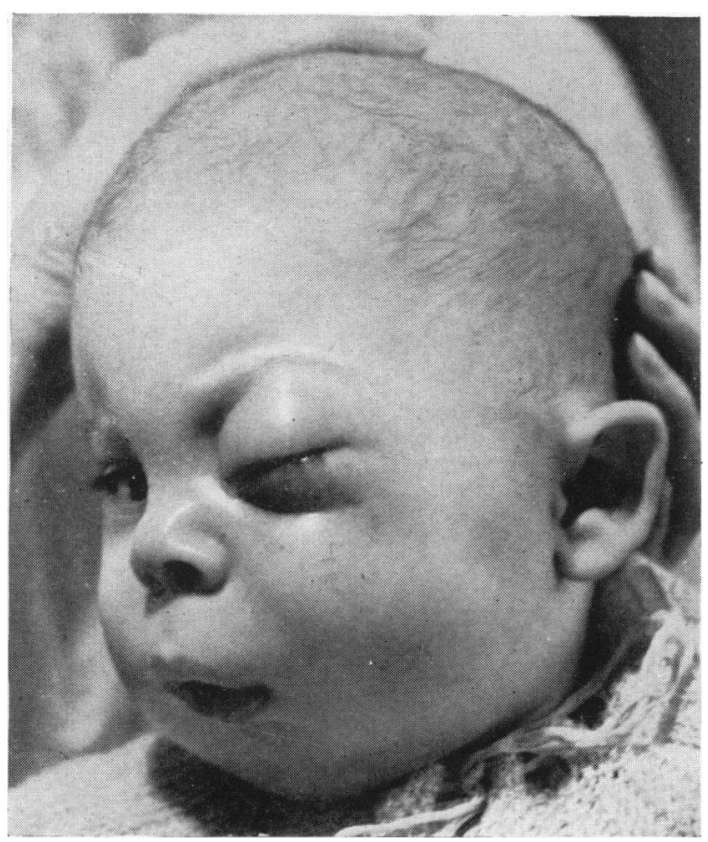

(a)-Case 4. Condition on admission to hospital.

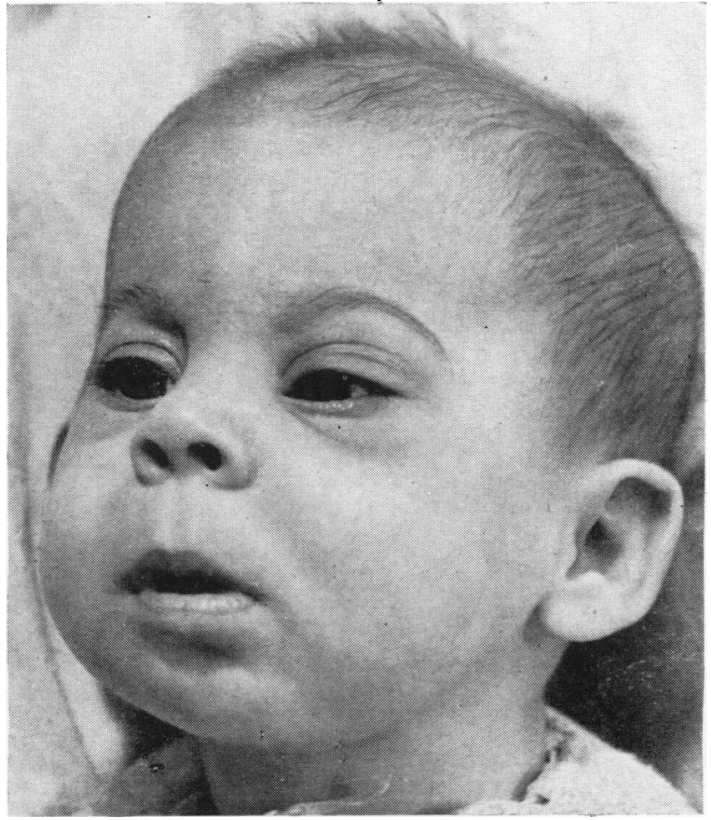

(b)-Case 4. Condition on discharge from hospital. 